\title{
PRODUÇÃO DE ÁCIDOS GRAXOS LIVRES A PARTIR DA HIDRÓLISE IMEDIATA DE ÓLEOS E GORDURAS RESIDUAIS EM ÁGUA SUBCRÍTICA: PRÉ-TRATAMENTO E CARACTERIZAÇÃO DO OGR
}

\author{
L. P. TORALLES ${ }^{1}$, C.T. ALVES ${ }^{1}$, E.A. TORRES ${ }^{1}$ e S.A.B. VIEIRA DE MELO ${ }^{1}$ \\ ${ }^{1}$ Universidade Federal da Bahia, Escola Politécnica, Programa de Engenharia Industrial \\ E-mail para contato: lucianatoralles@ gmail.com
}

\begin{abstract}
RESUMO - A hidrólise de óleos e gorduras residuais (OGR) em água subcrítica, para produzir ácidos graxos livres, seguida da esterificação destes, via catálise heterogênea, para produção de biodiesel, é uma alternativa promissora. A primeira etapa desse processo é o pré-tratamento e caracterização do OGR, objeto do presente estudo. O OGR foi prétratado e caracterizado quanto à sua composição de ácidos graxos usando ressonância magnética nuclear protônica $\left(\mathrm{RMN}^{+}\right)$, identificando-se o ácido linoleico como principal componente $(55 \%)$, em concordância com o óleo de soja refinado típico. Através da análise de $\mathrm{RMN}^{+}$foi possível calcular a massa molecular do OGR, cerca de $873 \mathrm{~g} / \mathrm{mol}$. A continuação desse estudo consistirá nos experimentos de hidrólise imediata para a obtenção de AGL's.
\end{abstract}

\section{INTRODUÇÃO}

Devido à maior demanda de energia, associada à uma crescente preocupação socioambiental com a emissão de gases de efeito estufa, a produção de biocombustíveis tem sido alvo de grande interesse para inovações tecnológicas no campo de energias renováveis (De Jong, 2013). Entre os biocombustíveis, o biodiesel é uma alternativa promissora para a substituição dos combustíveis fósseis, pois sua combustão libera quantidades menores de determinados poluentes atmosféricos, como hidrocarbonetos e monóxido de carbono (Janaun e Ellis, 2010). No entanto, apesar de ser considerado um combustível ambientalmente sustentável, a ampliação do consumo de biodiesel ainda depende da sua fonte de matéria-prima e de um maior desenvolvimento da sua tecnologia de produção (Atabani et al. 2012).

O biodiesel pode ser obtido a partir de diversas fontes renováveis que contenham ácidos graxos livres ou triglicerídeos, como óleos vegetais ou gordura animal (Marchetti et al. 2008). Após a utilização dos óleos vegetais, o reúso de óleos e gorduras residuais (OGR) impede que o óleo usado seja descartado inadequadamente, evitando contaminação do meio ambiente, podendo ser aproveitado como matéria prima na produção de biodiesel. Além disso, por se tratar de um óleo residual, o custo do OGR é significativamente menor que do óleo vegetal refinado, podendo levar à redução do custo operacional de produção de biodiesel (Yaakob et al. 2013). 


\section{9 a 22 de outubro de 2014 \\ Florianópolis/SC}

Hoje, há basicamente dois métodos para produção de biodiesel. O primeiro envolve a transesterificação dos triglicerídeos através do uso de metanol, com ou sem catalisador, produzindo biodiesel em uma única etapa (Saka e Kusdiana, 2001). O segundo método requer duas etapas: a hidrólise do óleo, produzindo ácidos graxos livres e glicerol, seguida da reação de esterificação dos ácidos graxos livres (Saka et al. 2006). Em ambos os métodos, existem alguns procedimentos diversos para obtenção do biodiesel, que visam atingir a especificação requerida juntamente com a redução dos custos operacionais, tais como reações não catalíticas ou catalíticas, com processos homogêneos ou heterogêneos (Hassan e Kalam, 2013). Recentemente, experimentos realizados em condições sub e/ou supercríticas, revelaram resultados promissores na produção deste biocombustível (Ju et al. 2013; Alves et al. 2013; Sawangkeaw et al. 2011; Maçaira et al. 2011).

A produção de biodiesel em um sistema de duas etapas apresenta diversas vantagens quando comparado com o método da transesterificação, incluindo menor tempo de reação e maior pureza do produto (Kusdiana e Saka, 2004). Ao se utilizar água a temperaturas entre seu ponto de ebulição (100 $\left.{ }^{\circ} \mathrm{C}\right)$ e seu ponto crítico $\left(374{ }^{\circ} \mathrm{C}\right)$, mantida no estado líquido sob pressão, alcança-se o seu estado subcrítico. Nesta condição, as propriedades da água se modificam, exibindo propriedades mais similares com as dos solventes orgânicos, pois o aumento da temperatura no estado líquido resulta em uma redução da constante dielétrica da água e consequente aumento da sua solubilidade no óleo (Carr et al. 2011). Assim, a água em condições subcríticas atua na hidrólise como reagente e solvente da reação (Pinto e Lancas, 2006; King et al. 1999). Também já foi relatado que a reação de hidrólise de óleos vegetais é auto catalisada pelos ácidos graxos livres formados, que agem como catalisadores ácidos, acelerando sua própria reação (Alenezi et al. 2010; Milliren et al. 2013).

O presente trabalho tem como objetivo o pré-tratamento e caracterização o OGR a ser utilizado na obtenção de ácidos graxos livres para produção de biodiesel, utilizando apenas água em estado subcrítico na reação de hidrólise. Através do aproveitamento do OGR, este trabalho contribui para o desenvolvimento de uma nova rota de produção de biodiesel ambientalmente sustentável.

\section{PARTE EXPERIMENTAL}

\subsection{Materiais e Equipamentos}

O óleo e gordura residual foi proveniente das regiões metropolitanas de Salvador, sendo recebido como doação no Laboratório de Energia e Gás da Universidade Federal da Bahia. O OGR foi pré-tratado e caracterizado antes da reação de hidrólise. Água destilada e deionizada foi utilizada nas etapas de pré-tratamento e na hidrólise do óleo. Também foram utilizados hidróxido de potássio (85\%) e hidróxido de sódio (97\%), fornecidos pela Fmaia, bem como solução de éter etílico e álcool etílico, ambos P.A., do mesmo fornecedor, na etapa de caracterização e pré-tratamento do OGR. As reações de hidrólise foram conduzidas no reator Parr com capacidade de 1 L. Este reator possui controladores de pressão, temperatura e velocidade de agitação. 


\subsection{Pré-tratamento e Caracterização do OGR}

O pré-tratamento do óleo consistiu de duas etapas: filtração e neutralização. A etapa de filtração teve o objetivo de remover materiais sólidos, seguida pela etapa de neutralização, realizada para a eliminação de ácidos graxos livres e materiais saponificáveis. Nesta última etapa, foi utilizado hidróxido de sódio e feitas lavagens cuidadosas com água destilada para remoção de impurezas. A Figura 1 mostra a etapa de lavagem do óleo após sua neutralização.

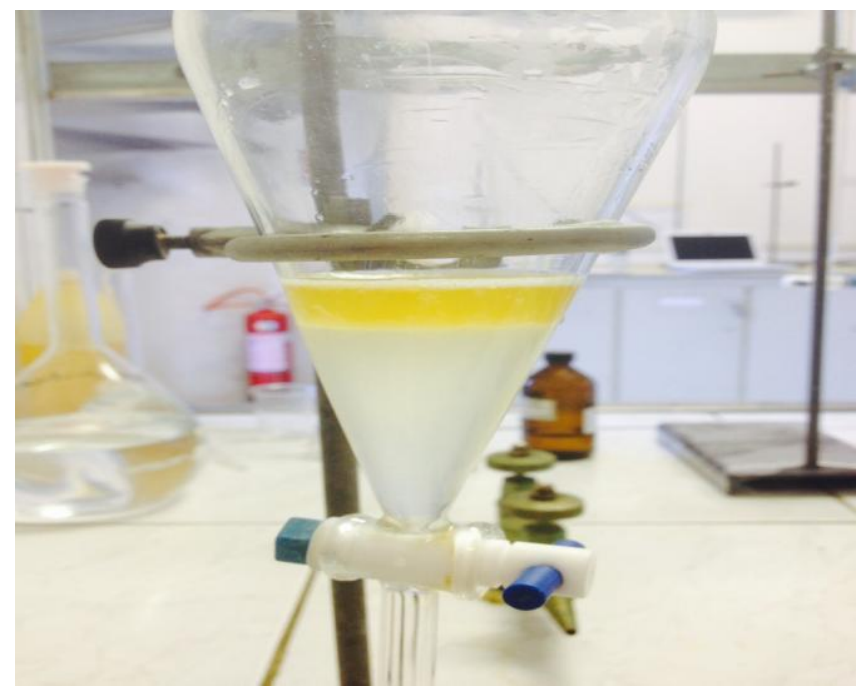

Figura 1 - Lavagem do OGR no pré-tratamento

O OGR foi caracterizado quanto ao seu índice de acidez (I.A.) antes e após o pré-tratamento. O I.A. foi calculado a partir da adição controlada de hidróxido de potássio até a neutralização do óleo, mostrada através de um indicador de $\mathrm{pH}$. Sua viscosidade cinemática foi medida no viscosímetro SCHOTT CT 52. As análises elementares de ácidos graxos (CHNOS), ressonância magnética nuclear protônica $\left(\mathrm{RMN}^{+}\right)$e cromatografia gasosa também foram realizadas com o objetivo de definir os principais componentes do OGR e calcular a sua massa molecular. Os resultados obtidos foram comparados com os de uma amostra de óleo de soja refinado, previamente analisada.

\subsection{Hidrólise do OGR}

As reações de hidrólise do OGR foram realizadas na próxima etapa do processo utilizando o reator Parr. O planejamento de experimentos realizado indicou as temperaturas entre 250 e $350{ }^{\circ} \mathrm{C}$. O tempo de residência variou entre 20 e $40 \mathrm{~min}$ com análise de amostras a cada $10 \mathrm{~min}$. Foi utilizado um excesso de água para favorecer a reação, sendo aplicada uma proporção experimental entre água e óleo de 4:1 e 6:1 em volume. A pressão foi mantida constante (18 MPa) em todos os experimentos, com uma velocidade de agitação fixa em $700 \mathrm{rpm}$. A Figura 2 mostra o fluxograma esquemático do processo. 


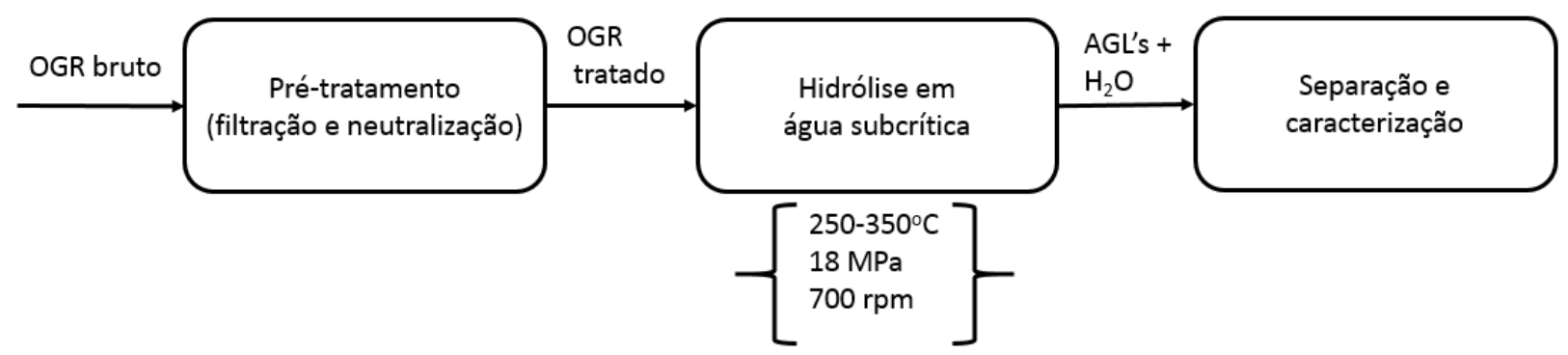

Figura 2 - Fluxograma do processo.

Neste processo, o óleo e a água foram adicionados ao reator e mantidos em descanso até que o reator atingisse a temperatura e pressão requeridas. A reação foi iniciada juntamente com a agitação e, após o período reacional, a amostra foi resfriada e posteriormente, removida do reator. A água, contendo glicerol, foi separada da fase oleosa, que contém ácidos graxos livres, através de decantação, permitindo a caracterização dos produtos obtidos.

\subsection{Caracterização de ácidos graxos e glicerol}

O rendimento da reação de hidrólise foi investigado através das análises de CG, juntamente com a análise elementar, para definir as composições e respectivas modificações nos ácidos graxos livres obtidos. Similarmente, também foi feita a avalição do rendimento do glicerol nessa reação de hidrólise. A cromatografia gasosa (CG Varian 3800A) é equipada com detector de ionização de chama (FID), onde será utilizada uma coluna de VF-5HT Ultimetal (30 $\mathrm{m}$ x 0,32 $\mathrm{mm}$ x $0,10 \mu \mathrm{m}$ ) para a separação cromatográfica e Hélio $(3,5 \mathrm{~mL} / \mathrm{min})$ como gás de arraste. A programação da temperatura de análise foi iniciada a $100{ }^{\circ} \mathrm{C}$, com um aumento para $250{ }^{\circ} \mathrm{C}$ em uma rampa de $15{ }^{\circ} \mathrm{C} / \mathrm{min}$, seguido por um aumento de $250{ }^{\circ} \mathrm{C}$ para $360{ }^{\circ} \mathrm{C}$, a uma velocidade de $8{ }^{\circ} \mathrm{C} / \mathrm{min}$, quando permaneceu a $360{ }^{\circ} \mathrm{C}$ por 10 min.

\section{RESULTADOS E DISCUSSÃO}

\subsection{Caracterização do OGR}

O OGR proveniente de estabelecimentos das regiões metropolitanas de Salvador-Ba apresentou um índice de acidez médio de 7,9 mg KOH/g de óleo antes do seu pré-tratamento. Para ser submetido à reação de hidrólise sem as interferências conhecidas, o óleo foi filtrado e neutralizado, resultando em uma perda de aproximadamente 15\% da matéria-prima. Após o pré-tratamento, seu índice de acidez foi avaliado novamente e comparado ao índice de acidez do óleo de soja refinado, da marca Sinhá, adquirido pelo Laboratório de Energia e Gás. Os resultados obtidos estão descritos na Tabela 1 , onde se verifica que o pré-tratamento atingiu uma redução superior a $90 \%$ dos materiais saponificáveis presentes na amostra. 
Tabela 1 - Índice de acidez (I.A.)

\begin{tabular}{|c|c|c|c|}
\hline Experimento & OGR bruto & OGR tratado & Óleo de Soja Refinado \\
\hline 1 & 7.61 & 0.62 & 0.84 \\
\hline 2 & 8.18 & 0.56 & 0.70 \\
\hline 3 & 7.85 & 0.59 & 0.65 \\
\hline $\begin{array}{c}\text { Média do I.A. } \\
\text { (mg KOH/g óleo) }\end{array}$ & $\mathbf{7 , 9}$ & $\mathbf{0 , 6}$ & $\mathbf{0 , 7}$ \\
\hline
\end{tabular}

As amostras de OGR também foram analisadas quanto à sua viscosidade cinemática no viscosímetro SCHOTT CT 52 a $40{ }^{\circ} \mathrm{C}$. A viscosidade foi calculada a partir da velocidade do escoamento do óleo dentro do capilar $\mathrm{n}^{\circ} 200$, por onde foi determinado o valor de 34,6 cSt, próximo ao valor do óleo de soja testado $(39,4 \mathrm{cSt})$. A análise elementar dos ácidos graxos foi realizada para definir a composição do OGR e calcular sua massa molecular. A Tabela 2 apresenta os resultados gerados por essa análise, onde se observa que o ácido linoleico, o ácido oleico e o ácido palmítico são os principais componentes do OGR. A partir do resultado obtido, a massa molecular calculada foi de $873 \mathrm{~g} / \mathrm{mol}$ do OGR.

Tabela 2 - Análise da Composição dos Ácidos Graxos do OGR

\begin{tabular}{|c|c|c|c|c|}
\hline Ácidos Graxos & Nomenclatura & Amostra $01(\%)$ & Amostra $02(\%)$ & Óleo de Soja (\%) \\
\hline C16:0 & Ácido Palmítico & 11,33 & 11,48 & 12,50 \\
\hline C18:0 & Ácido Esteárico & 3,53 & 3,53 & 0,65 \\
\hline 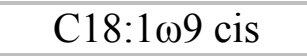 & Ácido Oleíco & 22,71 & 21,73 & 27,81 \\
\hline C18:1 $\omega 9$ trans & Ácido Eláidico & 1,49 & 1,43 & - \\
\hline $\mathrm{C} 18: 2 \omega 6 \mathrm{cis}$ & Ácido Linoléico & 54,82 & 55,67 & 54,19 \\
\hline C18:2 $\omega 6$ trans & Ácido Linolelaídico & 0,18 & 0,16 & - \\
\hline $\mathrm{C} 18: 3 \omega 3$ & Ácido Linolênico & 5.62 & 6 & 4,67 \\
\hline C20:0 & Ácido Eicosanóico & 0,32 & 0 & - \\
\hline
\end{tabular}

A análise de $\mathrm{RMN}^{+}$do OGR mostrou resultados similares ao espectro apresentado em publicações anteriores (Geris et al. 2007, Candeia, 2008). Portanto, a partir do $\mathrm{RMN}^{+}$, foi confirmada a presença de grupos característicos do óleo de soja. Os picos mostrados na Figura 3 foram identificados a partir dos seus conjuntos de sinais, conforme apresentado na Tabela 3. 


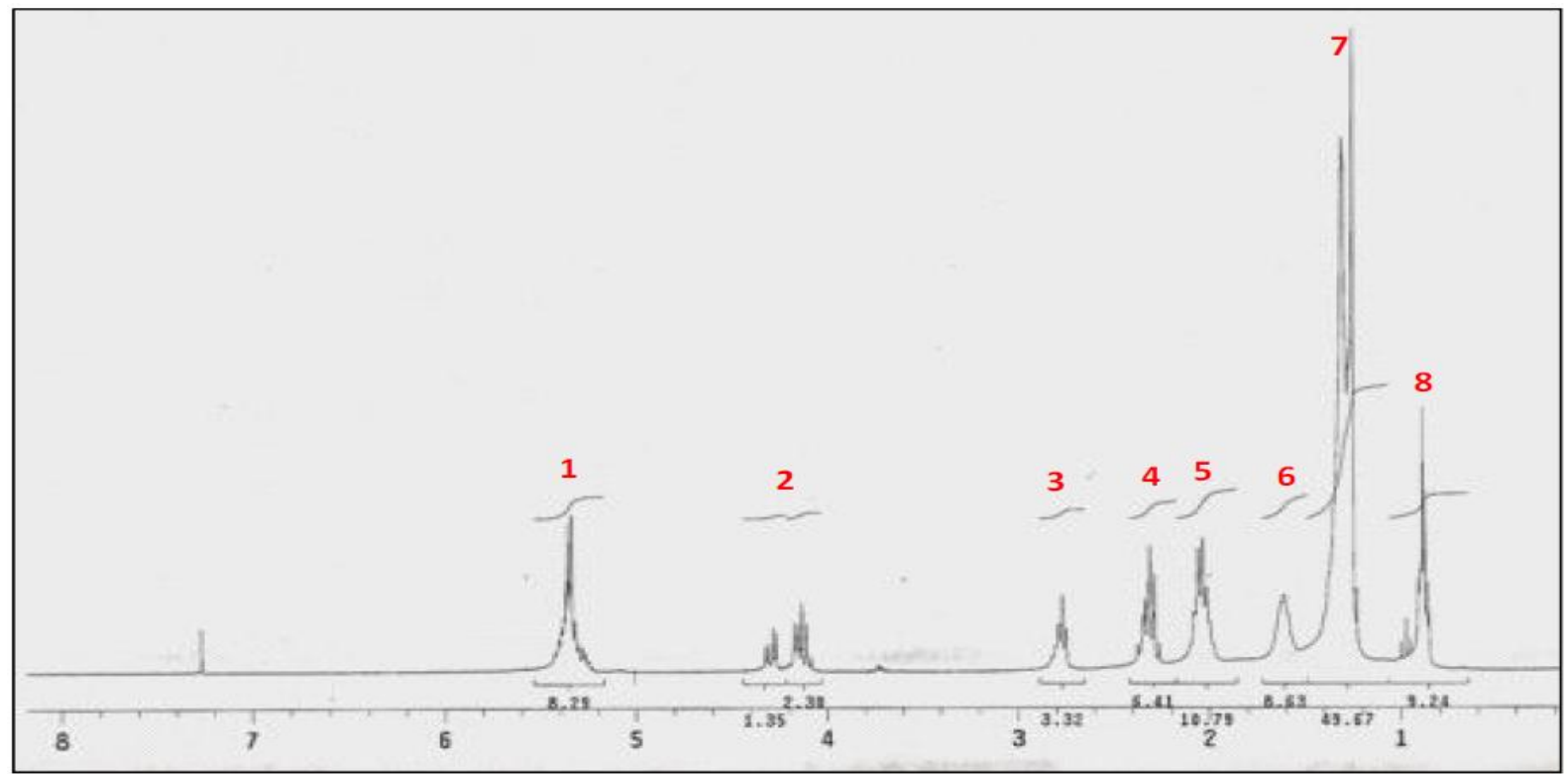

Figura 3 - Análise de $\mathrm{RMN}^{+}$do OGR.

Tabela 3 - Identificação dos picos detectados na análise de $\mathrm{RMN}^{+}$do OGR (Adaptado de Candeia, 2008)

\begin{tabular}{|c|c|c|c|}
\hline & Tipos de hidrogênios & Estrutura química & Sinal \\
\hline & & $-\mathrm{CH}=\mathrm{CH}-$ & \\
\hline 1 & $\begin{array}{l}\text { hidrogênios de olefina } \\
\text { hidrogênios metino da glicerina }\end{array}$ & CH-CO $\begin{array}{c}\mathrm{H}_{2}-\mathrm{CO}_{2} \\
\mathrm{CH}-\mathrm{CO}_{2}-\mathrm{CO}_{2}\end{array}$ & $5,20-5,40$ \\
\hline 2 & hidrogênios metilenos da glicerina & $\mathrm{CH}_{2}-\mathrm{CO}_{2} \mathrm{H}-\mathrm{CO}_{2}$ & $4,15-4,30$ \\
\hline 3 & hidrogênios dialilmetilênicos & $-\mathrm{CH}=\mathrm{CH}-\mathrm{CH}_{2}-\mathrm{CH}=\mathrm{CH}-$ & $3,60-3,80$ \\
\hline 4 & grupos metilenos $\alpha$-carboxilicos & $-\left(\mathrm{CH}_{2}\right)_{n}-\mathrm{CH}_{2}-\mathrm{CO}_{2}$ & $2,20-2,40$ \\
\hline 5 & grupos vizinhos aos carbonos insaturados & $-\mathrm{CH}=\mathrm{CH}-\mathrm{CH}_{2}-\left(\mathrm{CH}_{2}\right)_{n}-$ & $1,85-1,65$ \\
\hline 6 & $\begin{array}{l}\text { grupos metilenos vzinhos aos grupos } \mathrm{CH}_{2} \\
\text { alilicos }\end{array}$ & $\mathrm{CH}=\mathrm{CH}-\mathrm{CH}_{2}-\mathrm{CH}_{2}-\left(\mathrm{CH}_{2}\right)_{n}-\mathrm{CH}_{3}$ & $1,45-1,65$ \\
\hline 7 & $\begin{array}{l}\text { grupos metilenos vizinhos aos átomos de } \\
\text { carbono }\end{array}$ & $\mathrm{CH}_{2}-\left(\mathrm{CH}_{2}\right)_{n}-\mathrm{CH}_{3}$ & $1,15-1,40$ \\
\hline 8 & grupos metilicos terminais & $-\left(\mathrm{CH}_{2}\right)_{n}-\mathrm{CH}_{3}$ & $0,80-1,0$ \\
\hline
\end{tabular}


Para maior precisão, a análise elementar e a análise de $\mathrm{RMN}^{+}$do OGR foram comparadas à do óleo hidrolisado. Essa comparação foi utilizada para confirmar as modificações na molécula do óleo e a geração de ácidos graxos livres.

\section{CONCLUSÃO}

O presente trabalho apresentou os resultados dos experimentos de pré-tratamento do OGR e suas respectivas caracterizações de índices de acidez do OGR bruto e tratado. Foram realizadas as análises de viscosidade cinemática, análises elementares de ácidos graxos (CHNOS) e ressonância magnética nuclear protônica $\left(\mathrm{RMN}^{+}\right)$. O OGR apresentou características similares às de estudos anteriores, com valores de viscosidade cinemática e índice de acidez próximos aos do óleo de soja refinado após seu pré-tratamento. $\mathrm{Na}$ etapa seguinte deste estudo, o óleo tratado foi submetido à hidrólise com água subcrítica para geração de ácidos graxos livres destinados à produção de biodiesel.

\section{REFERÊNCIAS}

ALENEZI, R.; BAIG, M.; WANG, J.; SANTOS, R.C.D.; LEEKE, G. Continuous Flow Hydrolysis of Sunflower Oil for Biodiesel. Energy Sources Part A Recovery Utilization and Environmental Effects. v.32, p. 460-468, 2010.

ALVES, C.T.; ANDRADE, H.M.C.; VIEIRA de MELO, S.A.B.; TORRES, E.A. Transesterification of waste frying oil using a zinc aluminate catalyst, Fuel Process. Technol v. 106, p. 102-107, 2013.

ATABANI A.E.; SILITONGA, A.S.; BADRUDDIN, I. A.; MAHLIA, T.M.I.; MASJUKI, H.H.; MEKHILEF, S. A comprehensive review on biodiesel as an alternative energy resource and its characteristics. Renewable and Sustainable Energy Reviews. v. 16, n. 4. p. 2070-2093, 2012.

CANDEIA, R. Biodiesel de Soja: Síntese, Degradação e Misturas Binárias. 2008. 132f. Tese de Doutorado (Departamento de Químiza). Universidade Federal da Paraíba, João Pessoa, 2008.

CARR A.G, MAMMUCARI R., FOSTER N.R. A review of subcritical water as a solvent and its utilisation for the processing of hydrophobic organic compounds. Chem. Eng. J. v.172 .p.1-17, 2011

DE JONG, Pieter. Economic, technical and environmental analysis of renewable and non-renewable electricity generation technologies in Brazil. Dissertação (Mestrado em Engenharia Industrial). Escola Politécnica, Universidade Federal da Bahia, Salvador, 2013.

GERIS, R.; dos SANTOS, N.A.C.; AMARAL, B.A.; MAIA, I.S.; CASTRO, V.D.; CARVALHO, J.R.M. Biodiesel from soybean oil - experimental procedure of transesterification for organic chemistry laboratories. Química Nova, v. 30, n. 5, p. 1369-1373, 2007.

HASSAN, M., KALAM, M. An Overview of Biofuel as a Renewable Energy Source: Development and Challenges. Procedia Eng. v. 56. p. 39-53, 2013.

JANAUN, J. e ELLIS, N. Perspectives on biodiesel as a sustainable fuel. Renewable and Sustainable Energy Reviews. v. 14, n. 4, p. 1312-1320, 2010.

JU,Y.; HUYNH, L. H.; TSIGIE, Y.A.; HO, Q. Synthesis of biodiesel in subcritical water and methanol. Fuel, v.105, p.266-271, 2013. 
KING, J.W., HOLLIDAY, R.L. and LIST, G.R. Hydrolysis of soybean oil in a subcritical water flow reactor. Green Chem, v.1. p. 261-264, 1999.

KUSDIANA, D. e SAKA, S. Two-step preparation for catalyst-free biodiesel fuel production hydrolysis and methyl esterification. Appl Biochem Biotechnol. 781. p. 113-116, 2004

MAÇAIRA, J.; SANTANA, A.; RECASENS, F.; LARRAYOZ, M.A. Biodiesel production using supercritical methanol/carbon dioxide ixtures in a continuous reactor. Fuel. v. 90. n.6. p. 2280-2288, 2011.

MARCHETTI. J.M., MIGUEL, V.U., ERRAZU, A.F. Techno-economic study of different alternatives for biodiesel production, Fuel Proc Tech. v.89 p. 740-748, 2008.

MILLIREN, A.L., WISSINGER, J.C., GOTTUMUKALA, V. SCHALL, C.A. Kinetics of soybean oil hydrolysis in subcritical water. Fuel. v. 108. p. 277-281, 2013.

PINTO, J.S.S. e LANCAS, F.M. Hydrolysis of corn oil using subcritical water. J Brazil Chem Soc, v. 17, p.85-89, 2006.

SAKA, S., KUSDIANA, D. MINAMI, E. Non-catalytic biodiesel fuel production with supercritical methanol technologies. J. of Scientific \& Ind. Research. v. 65. p. 420-425, 2006.

SAKA, S. e KUSDIANA, D., Biodiesel fuel from rapeseed oil as prepared in supercritical methanol. Fuel. v. 80. p. 225-231, 2001.

SAWANGKEAW, R.; TEERAVITUD, S.; BUNYAKIAT, K.; NGAMPRASERTSITH, S. Biofuel production from palm oil with supercritical alcohols: Effects of the alcohol to oil molar ratios on the biofuel chemical composition and properties. Bioresource Technology, v.102. n. 22. p. 10704-10710, 2011.

YAAKOB, Z., MOHAMMAD, M., ALHERBAWI, M., ALAM Z., SOPIAN, K. Overview of the production of biodiesel from Waste cooking oil. Renewable and Sustainable Energy Reviews. v.18. p. 184-193, 2013. 